\title{
ANOMALOUS GALVANOMAGNETIC EFFECTS IN InSb THIN FILMS WITH SUPERCONDUCTING LEAD INCLUSIONS*
}

\author{
O.A. MiRonov, O.N. NashcheKINA \\ Institute of Radiophysics and Electronics , Acad. Sci. Ukraine \\ Acad. Proscura 12, 310085 Kharkov, Ukraine \\ M. OSZWALDOWSKI AND T. BERUS \\ Instytut Fizyki, Politechnika Poznańska, Piotrowo 3, 60-965 Poznań, Poland \\ (Received May 29, 1992, in final form November 2, 1992)
}

\begin{abstract}
We report on the first experimental observations of an anomalous increase in the resistance of a semiconductor-superconductor contact in the vicinity of the critical temperature $T_{\mathrm{c}}$. The effect is found in lead-doped $\mathrm{InSb}$ thin films having inclusions of lead of $1-3 \mu \mathrm{m}$ in diameter: The observed effect can be a model that explains similar resistance increase observed sometimes in the high $T_{c}$ superconductors.
\end{abstract}

PACS numbers: 72.2 0.My, 74.50.+r

\section{Sample preparation and characteristics}

Lead-doped InSb thin films, 2-3 $\mu \mathrm{m}$ thick, were obtained by the coevaporation of InSb and lead from separate sources on ceramic substrates. The film preparation method was essentially the same as that for tin-doped InSb films [1]. The lead content in the films was varied by a change of the Pb-evaporation-source temperature between $950 \mathrm{~K}$ and $1250 \mathrm{~K}$. Several films were obtained in one evaporation process. A part of the films from each evaporation run was regrown from the melt by a microzone-melting procedure [1].

The electrical properties of the films were investigated with the IIall effect measurements. The morphology and chemical composition of the films were studied with optical microscope observations and selective etchings accompanied with chemical analysis. Further detailed studies of the morphology and chemical composition were carried out with a scanning electron microscope and X-ray microanalyser [1]. The investigation results can be summarized as follows. With-

*This paper was presented at the XXI International School on Physics of Semiconducting Compounds, Jaszowiec (Poland), 1992. 
out any intentional doping, the electron concentration in the films was $n \approx 6 \times$ $10^{16} \mathrm{~cm}^{-3}$. The electron mobility in the vapour grown (VG) films was $\mu \leq$ $0.6 \mathrm{~m}^{2} / \mathrm{Vs}$, and in the melt-regrown (MR) $\mu \leq 3 \mathrm{~m}^{2} / \mathrm{Vs}$. The Pb-doped films, both VG and MR, obtained with a $\mathrm{Pb}$ source temperature $T_{\mathrm{s}}<1000 \mathrm{~K}$ show electrical properties close to those of undoped $\mathrm{InSb}$ films. For $T_{s}>1000 \mathrm{~K}$ the films have a two-phase character, i.e. they contain metallic inclusions in the InSb matrix. In the VG films the inclusions have more regular form and are smaller in diameter but larger in number. The diameter and the density of the inclusions depend on the amount of the evaporated lead; typically, the diameter of the inclusion in the VG films is several $\mu \mathrm{m}$, whereas that in the MR films is of the order of $50 \mu \mathrm{m}$.

Figure 1a shows a typical fragment of a surface topography of a VG film with the inclusions in the form of irregular droplets. Figure $1 \mathrm{~b}$ shows that the inclusions are chemically composed mainly of lead. The electron concentrations in the
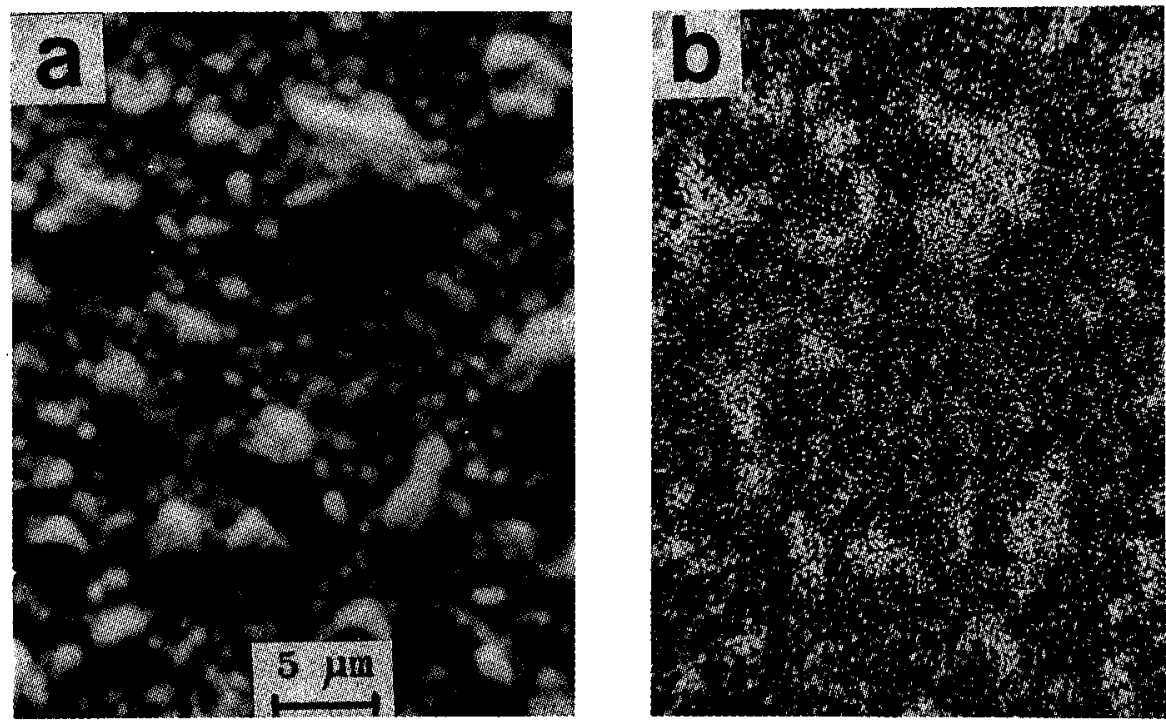

Fig. 1. Surface appearance of a VG InSb film with lead inclusions as obtained with a scanning electron microscope and $X$-ray analyser; (a) surface topography seen in the secondary-electron image, (b) the same area seen in the characteristic X-ray radiation of lead (the irregular white spots are the lead inclusions).

two-phase films, as formally deduced from the Hall coefficient $R_{\mathrm{H}}$, are considerably higher. However, when the inclusions are selectively etched from the films, the measured concentrations are close to those met in the single-phase films, i.e. they become smaller. This shows that the inclusions can very effectively short-circuit the Hall voltage, especially in the MR films. It can also be concluded that the lead has no or little effect on the donor concentration of the films, which seems to confirm that the lead is a neutral impurity in InSb [2]. 


\section{Galvanomagnetic properties}

The galvanomagnetic properties of the single-phase, $\mathrm{Pb}$-doped InSb films for $T>4 \mathrm{~K}$ are the same as those of the undoped films, whereas the properties of the two-phase films are affected by the short-circuiting effects of the lead inclusions. The typical temperature dependence of $R_{\mathrm{H}}$ and the conductivity $\sigma$ of the two-phase films is shown in Fig. 2. In Fig. 2a the dependence is shown for a sample $A$ which is a MR film, and in Fig. 2b for samples $B$ and $C$ which are VG films. The samples $A$ and $B$ were obtained in the same evaporation run.

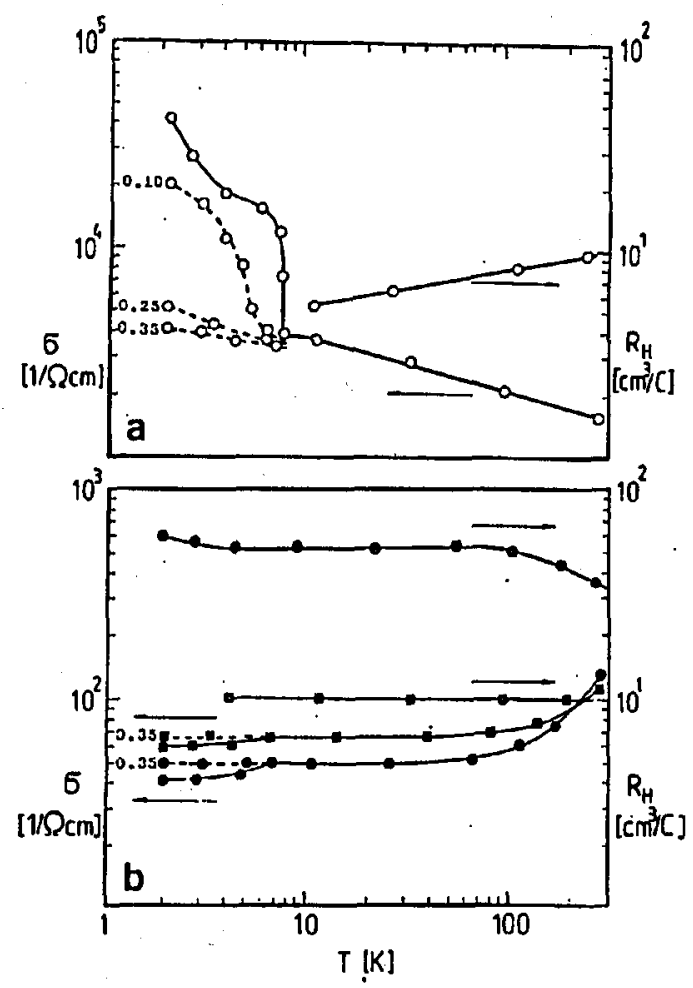

Fig. 2. Temperature dependence of the Hall coefficient $R_{\mathrm{H}}$ and conductivity $\sigma$ of the two-phase InSb-Pb thin films; (a) for the melt-regrown film $A$, (b) for the as-vapour-grown films $B(\bullet)$ and $C(\bullet)$. The dotted curves are the dependence of $\sigma$ in a magnetic field $B$. The values of $B$, in teslas, are given by the numbers at the curves.

The small $R_{\mathrm{H}}$ and its increase with $T$, as well as the large $\sigma$ and its linear decrease with $T$ observed in the sample $A$ show that their electrical properties are governed by the lead inclusions. The most pronounced short-circuiting effect of the inclusions takes place at $T<7 \mathrm{~K}$, when the inclusions become superconducting. The effect of the transition to superconductivity can entirely be suppressed by a magnetic field (the discontinuity observed in $\sigma$ is due to the magnetoresistance). As seen in Fig. 2b, the behavior of the samples $B$ and $C$ is quite different. Above 
$7 \mathrm{~K}$ their temperature dependence of $R_{\mathrm{H}}$ and $\sigma$ is close to the one met in the single-phase samples. This shows that the short-circuiting effect of the inclusions is less effective in the VG films. The most interesting is the decrease in $\sigma$ below $7 \mathrm{~K}$ which can be entirely suppressed by a magnetic field. This means that the superconductive inclusions can act as effective scattering centers. An increase in the resistance of a semiconductor-normal (S-N) boundary has been predicted by Kadigrobov [3]. The effect is due to scattering of a part of the electrons ("the gliding electrons") by an S-N boundary. The theory predicts a higher decrease in the resistance in the samples in which electrons have a lower Fermi energy. This is observed in the present case, as the sample $C$ has a smaller $n$, and thereby a lower Fermi energy. The magnitude of the effect is, however, in disagreement with the theory. The observed relative resistivity change is of the order 0.1 , whereas the theory predicts a change at least three orders of magnitude smaller.

The observed decrease in the resistance of samples having small semiconducting clusters can be useful in the explanation of similar resistance increase observed sometimes at $T_{\mathrm{c}}$ in the high- $T_{\mathrm{c}}$ superconductors.

\section{References}

[1] M. Oszwałdowski, T. Berus, Thin Solid Films 172, 71 (1989).

[2] O. Madelung, Physics of Semiconducting Compounds, Mir, Moscow 1967, p. 262 (in Russian).

[3] A.M. Kadigrobov, Sov. J. Low Temp. Phys. 14, 427 (1988). 